\title{
Auf ein Neues: Endarteriektomie versus Stenting der A. carotis interna
}

Die Restenoserate nach Stenting oder Endarteriektomie wird in Studien sehr unterschiedlich angegeben. Der Grund dafür sind wahrscheinlich unterschiedliche Ultraschallkriterien für eine Stenose.

\begin{abstract}
$D^{-1}$ „Carotid Revascularization Endarterectomy versus Stenting Trial“ (CREST) ist seit einigen Jahren publiziert, sie konnte prospektiv und randomisiert zeigen, dass der primäre Endpunkt von Schlaganfall, Herzinfarkt und periprozeduralem Tod zwischen der Endarteriektomie und dem Stenting nicht signifikant unterschiedlich ist. Dies galt sowohl für symptomatische als auch für asymptomatische Stenosen. Ein sekundärer Endpunkt von CREST war das Auftreten von Restenosen oder Verschlüssen. Dies wurde in der vorliegenden Studie analysiert. Patienten mit einer Stenose der A. carotis interna $(>70 \%$ nach Ultraschallkriterien), die asymptomatisch waren oder eine TIA, eine Amaurosis fugax oder einen kleineren Schlaganfall erlitten hatten, wurden in CREST eingeschlossen (zwischen 2000 und 2008 in 117 Zentren in den USA und Kanada). Der o.g. sekundäre Endpunkt wurde nach zwei Jahren mittels Ultraschalluntersuchung bestimmt. Hierzu musste eine zumindest $70 \%$-Stenose nachgewiesen werden. 2.191 Patienten wurden eingeschlossen (1.086 mit einem Stenting und 1.105 mit einer Endarteriektomie). Nach zwei Jahren hatten $58 \mathrm{~Pa}$ tienten mit Stenting und 62 Patienten mit Endarteriektomie eine Restenose oder einen Verschluss $(p=0,58)$. Weibliches Geschlecht, Diabetes und Dyslipidämie waren unabhängige Prädiktoren einer Restenose oder eines Verschlusses.
\end{abstract}

Kommentar: Die Ergebnisse der CREST Studie belegen, dass die Rate von Restenosen oder Verschlüssen nach einem Stenting gering ist. Problematisch ist jedoch, dass diese Rate in anderen Studien mit Stenting $\operatorname{der} A$. carotis interna so unterschiedlich ist. Der Grund hierfür sind wahrscheinlich verschiedene Ultraschallkriterien für eine Stenose mit einem Grad von wenigstens $70 \%$. Es sei darauf hingewiesen, dass in der ähnlichen angelegten SPACE-Studie die Rate von Restenosen nach zwei Jahren in der Gruppe mit Stenting signifikant höher war als in der Gruppe mit Endarteriektomie

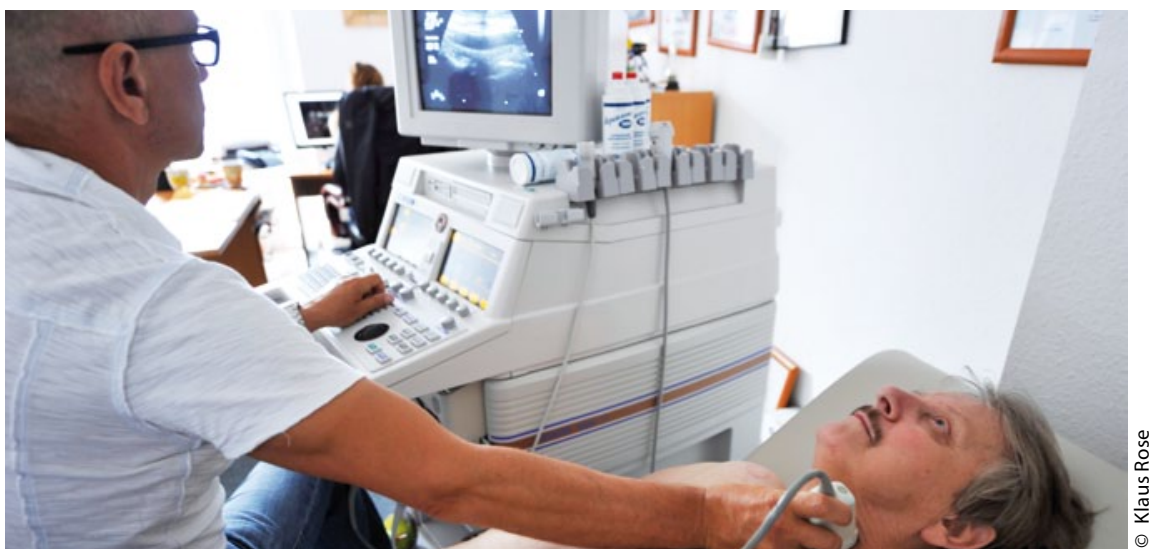

Ultraschallkontrolle der Arteria carotis

$(10,7 \%$ versus $4,6 \%)$. Wenn man als Grenze für eine höhergradige Stenose eine systolische Maximalgeschwindigkeit von $>2,1 \mathrm{~m} / \mathrm{s}$ zugrundelegt, zeigte sich auch in der CRESTStudie eine signifikant höhere Rate von Restenosen in der Gruppe mit Stenting. Außerdem konnte in der Studie nicht gezeigt werden, ob eine höhere Rate von Restenosen auch mit einer erhöhten Rate von Schlaganfällen einhergeht. Nur dies würde ja regelmäßige Ultraschallkontrollen nach einem Stenting sinnvoll machen. Eine weitere Schwäche dieser Analyse ist, dass nur für eine Nachbeobachtung von zwei Jahren hinreichend Daten vorliegen. Für den Langzeitverlauf können also keine Aussagen gemacht werden. So werden wir auch weiterhin auf Studien mit längeren Beobachtungszeiten (ca. 10 Jahre sollten es schon sein) und noch höherer Fallzahl (um zu beurteilen, ob eine Restenose auch das Risiko für einen ipsilateralen Schlaganfall erhöht) warten müssen, um zwischen Stenting und Endarteriektomie evidenzbasiert wählen zu können.

Professor Stefan Evers

Lal BK et al. ( CREST Investigators): Restenosis after carotid artery stenting and endarterectomy: a secondary analysis of CREST, a randomised controlled trial. Lancet Neurol 2012; 11: 755-63 